\title{
Arrival Trajectory Optimization for Passenger Aircraft using Genetic Algorithms
}

\author{
H. $\mathrm{Yu}^{*}$ and J. A. Mulder ${ }^{\dagger}$ \\ Delft Univeristy of Technology, Kluyverweg 1, 2629 HS Delft, The Netherlands.
}

\begin{abstract}
This work concentrates on the development of an optimization technique which is capable of minimizing the noise impact of an arriving aircraft by optimizing its flight trajectory. Actions needed from pilots to gradually establish the landing configuration are considered because it is expected that the pilot workload throughout this phase should be remained or even reduced compared with the current standard arrival procedures. Therefore, the conventional point-mass equations of motion are reformulated in such a way that the variations of the aerodynamic performance of the aircraft of different configurations can be easily taken into account. A set of independent state variables are chosen to be parameterized with Bernstein polynomials in order to convert the infinite-dimensional optimal control problem into a finite-dimensional parametric optimization problem. The number of awakenings is selected as the performance index and finally written into a function of the parameters introduced by the parameterization process. Genetic algorithms are employed to optimize these parameters within a search domain in order to minimize the number of awakenings while satisfying all constraints on both state and control variables. A number of numerical examples, for a Boeing 747-400 aircraft arriving at an airport with different population distribution situations, are provided to demonstrate the feasibility and effectiveness of the proposed optimization technique. Without loss of generality, this particular technique is also able to deal with a departing aircraft since most of the models are built into replaceable modules.
\end{abstract}

\section{Nomenclature}

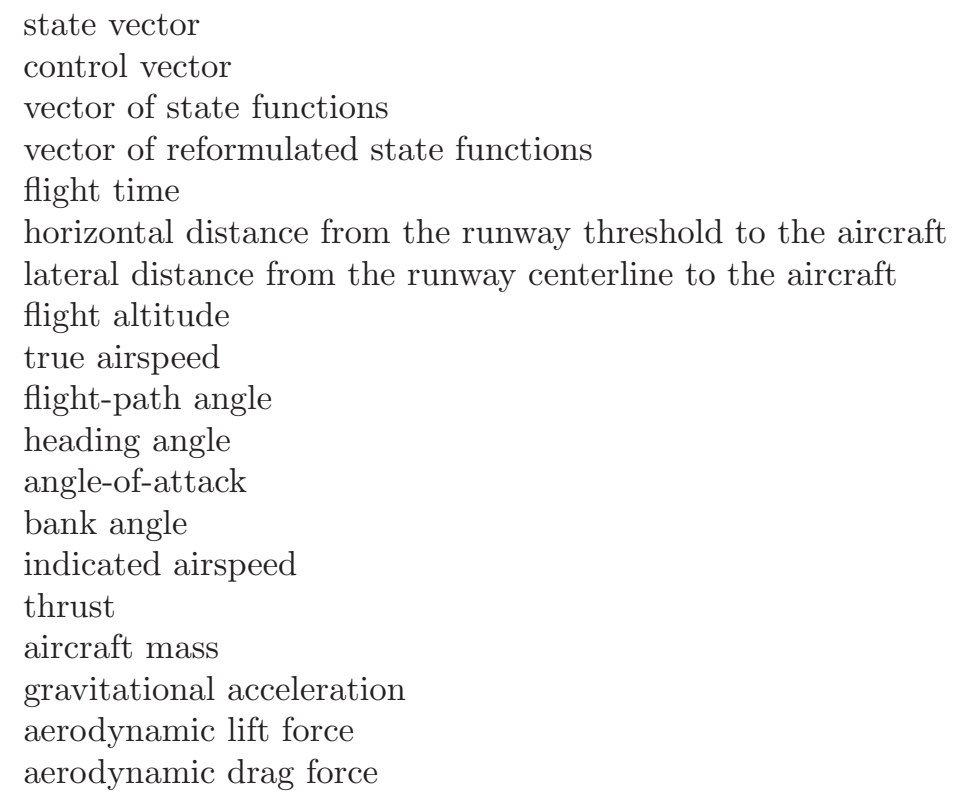

*Ph.D student, Control\&Operation Division, Faculty of Aerospace Engineering.

$\dagger$ Professor, Control\&Operation Division, Faculty of Aerospace Engineering. 
$C_{L} \quad$ aerodynamic lift coefficient

$C_{D}$ aerodynamic drag coefficient

$m$ dimension of the state vector

$r \quad$ dimension of the control vector

$B \quad$ Bernstein polynomials

$b \quad$ Bernstein basis polynomials

$\tau \quad$ independent variable in Bernstein polynomials

$J \quad$ cost function

Subscript

$i \quad$ initial

$f \quad$ final

Superscript

* optimal

\section{Introduction}

Trajectory optimization for passenger aircraft has been attracting wide attention because noise impact of civil aviation is one of the environmental issues with top priority in the field of air transport and aircraft operations. Community noise ${ }^{1}$ (also called environmental noise, residential noise, or domestic noise) is defined as the noise emitted form all sources except the noise at industrial workplaces. Although many airports were originally built at some distances from the communities they intended to serve, air traffic is counted as one of the main sources of community noise because of the ceaselessly increasing number of flight movements and the lack of space for the significantly expanding residential communities.

For the purpose of certificates, commercial aircraft have to meet certain standards on noise set by the International Civil Aviation Organization (ICAO). The latest and also strictest standard ${ }^{2}$ was introduced in October 2001 and revised in March 2002, which are applicable for certain types of aircraft currently in service and certificates submitted after January 1, 2006. In addition to setting a threshold for the entrance of the market, it is intended to gradually phase out those aircraft that do not fulfill the requirements. Next to this standard, airports also adopt noise-related operating restrictions on aircraft in order to reduce the noise impact on surrounding residential communities. For example, at Amsterdam Airport Schiphol (AAS) in The Netherlands, the number of flight movements within a certain time period is limited and noise levels on a daily basis are carefully measured and monitored to avoid the statutory limit values for noise impact being exceeded. Reconsidering the fact that there may be other tools than aircraft phasing-out and operating restrictions, the ICAO brought out the concept of "Balanced Approach" ${ }^{3}$ to address the noise issue of civil aviation in an environmentally responsive and economically responsible way in 2004. Encompassed are four principal elements as follows:

- reduction of noise at source;

- land-use planning and management;

- noise abatement operational procedures; and

- operating restrictions on aircraft.

In the present work, "land-use planning and management" is not under consideration and thus the distribution of residents around the airport is assumed to be fixed. Furthermore, it has been acknowledged by most of the ICAO contracting countries that "operating restrictions on aircraft" should not be taken as the first resort but after evaluating the benefits which can be gained from the other elements. Therefore, "reduction of noise at source" and "design of noise abatement operational procedures" become the only available options under this situation. Next to optimizing the shape of the aircraft and designing quiet engines, "reduction of noise at source" can also be achieved by flying the aircraft slower of cleaner configurations and reducing the thrust required from the engines. As a matter of fact, these two tricks are both considered when "noise abatement operational procedures" are designed. At present, quite a number of noise abatement operational procedures have been proposed and implemented in practical cases. For instance, noise abatement departure procedures designed by the ICAO are intended to minimize the total area impacted by the noise of a departing aircraft by climbing steeply or executing thrust cutbacks. As for arriving aircraft, procedures based on the Continuous Descent Approach ${ }^{4}$ (CDA) are employed to help reduce the noise impact when the air traffic is not too busy. Different from traditional step-down approaches, these CDA-based procedures reduce the 
noise perceived by the people on the ground via reducing the thrust to flight ideal and keeping the quieter aircraft higher for longer. By definition, possible operational procedures for noise abatement may include one or more of the following:

1. the use of noise preferential runways to direct the aircraft away from noise-sensitive areas;

2. the use of noise preferential flight paths to assist the aircraft in avoiding noise-sensitive areas; and

3. the use of noise abatement procedures designed to optimize the distribution of the noise on the ground. Corresponding to the third category, the objective of this work is the design of departure and arrival trajectories by optimizing the distribution of the noise on the ground to minimize the overall noise impact. By definition, minimizing (or maximizing) a certain performance index by choosing the flight trajectory is often referred to as "trajectory optimization". In this work, the optimization routine aims at generating environment-oriented optimal trajectories for an arriving aircraft whose noise impact is selected as the performance index to be minimized. Note that the performance index can be replaced by fuel consumption or else and the aircraft can be one that is departing, for which the principle of the optimization holds out but details need to be adjusted to adapt to the specific situation.

The optimization technique presented herein combines an aircraft model, the acoustic model of the selected aircraft, the population distribution around the scheduled airport, and then employs genetic algorithms to generate an optimal trajectory with respect to a preselected criterion. Considering a trajectory optimization problem, the movement of the aircraft is governed by the point-mass equations of motion. Conventionally, these equations include derivatives with respect to the flight time. In this work, however, state variables are selected in such a way that the equations of motion in the conventional form can be reformulated in to ones only involving derivatives with respect to the indicated airspeed. As a result, the variations of the aerodynamic performance of the arriving aircraft of different configurations can be easily taken into account. A well-established and highly successful approach to reach the solution of such a problem is to implement discretization on states or controls or both of them to yield a finite-dimensional parametric optimization problem ${ }^{5}$ which is computable using most of the existing algorithms. Approaches characterized by discretization followed by optimization are usually referred to as "direct methods", next to which are "indirect methods" as another category of feasible approaches ${ }^{6}$ for solving the general optimal control problems of infinite dimensions. In this work, state parameterization ${ }^{7,8}$ using Bernstein polynomials, considered as one direct method, are employed. A number of independent state variables are parameterized with the expectation that the remaining state and control variables can also be written into functions of the introduced parameters ${ }^{\mathrm{a}}$. Determined by the histories of some of the state and control variables, the performance index is also expected be written into a function of these parameters. Subsequently, genetic algorithms are employed to solve the newly formed finite-dimensional parametric optimization problem. Note the fact that it is very possible that optimal trajectories generated by optimization algorithms make the structure of the air traffic flow a lot more complex because the aerodynamic and acoustic performances of different aircraft are usually different and thus optimal trajectories for different aircraft may differs greatly. Meantime, it is also expected that advanced air transportation systems would impose less and weaker constraints on aircraft trajectories in the future. As a matter of fact, tools for communication, guidance and surveillance have been available or right under development so that trajectory optimization has been developing rather quickly.

In order to demonstrate the feasibility and effectiveness of the proposed optimization technique, a set of numerical examples are provided under a number of user-defined situations. In all cases, a Boeing 747-400 aircraft $^{9}$ is assumed to be a point-mass subject approaching the scheduled runway. Combining the NoisePower-Distance (NPD) tables of the selected aircraft and some of the formulas given in the Integrated Noise Model $^{10}$ (INM) yields a measure of calculating the noise levels at observing points located in the vicinity of the airport. Given these noise levels, one can finally calculate the number of awakenings generated by the arriving aircraft. With the number of awakenings as the performance index of the optimization, it can be said that the entire vicinity of the airport is under consideration and the optimal trajectory is indeed designed by optimizing the noise distribution on the ground. For the sake of simplicity, the population distribution is not from an actual airport but only built by the authors with a regular grid. For the purpose of comparison, however, there is more than one population distribution model presented in this work.

This paper is structured in the following way. In Section II, the authors first describe the reformulation of the point-mass equations of motion, as a result of which the derivatives on the left-hand side of the formulas become ones with respect to the indicated airspeed rather than the flight time. In addition, the authors

${ }^{a}$ It is possible that the analytical expressions of some of these remaining variables are not approachable. In this case, numerical integration has to be applied to obtain the histories of these variables. 
also introduce the relation between noise levels and the percent of awakenings in order to calculate the number of awakenings as the performance index. In Section III, state parameterization of independent state parameters using Bernstein polynomials and associated derivations are described step by step to obtain the performance index and those unparameterized state and control variables. In Section IV, models required in the optimization routine are presented with details. In Section V, a set of numerical examples are provided to demonstrate the usefulness of the proposed optimization technique. In the end, this work is concluded by Section VI.

\section{Problem Statement}

Dealing with a trajectory optimization problem, the aircraft is in general assumed to be a point-mass subject. Since the operating airspace is small enough and the flight time is short enough, the Earth is considered to be flat and non-rotational. Weather conditions, mainly wind, are not included in this work. It is assumed that all the forces acting on the aircraft go through its center of gravity and the angle between the engine thrust and the longitudinal axis of the aircraft is assume to be equal with zero. Additionally, the angle $\alpha$ is generally so small that following assumptions are applied:

$$
\left\{\begin{array}{l}
\sin \alpha=0 \\
\cos \alpha=1
\end{array} .\right.
$$

\section{A. Reformulation of Equations of Motion}

Denoted by $O X_{e} Y_{e} Z_{e}$, a local coordinate system on the ground (the surface of the Earth) is constructed in such a way that the origin $O$ locates at the threshold of the scheduled runway, with $O X_{e}$ lying on the ground plane pointing from the threshold to the other end of the runway, with $O Y_{e}$ perpendicular to $O X_{e}$ pointing to the left horizontally, and with $O Z_{e}$ pointing from the ground to the sky vertically. The coordinate system and all associated angles can be seen in Figure 1.

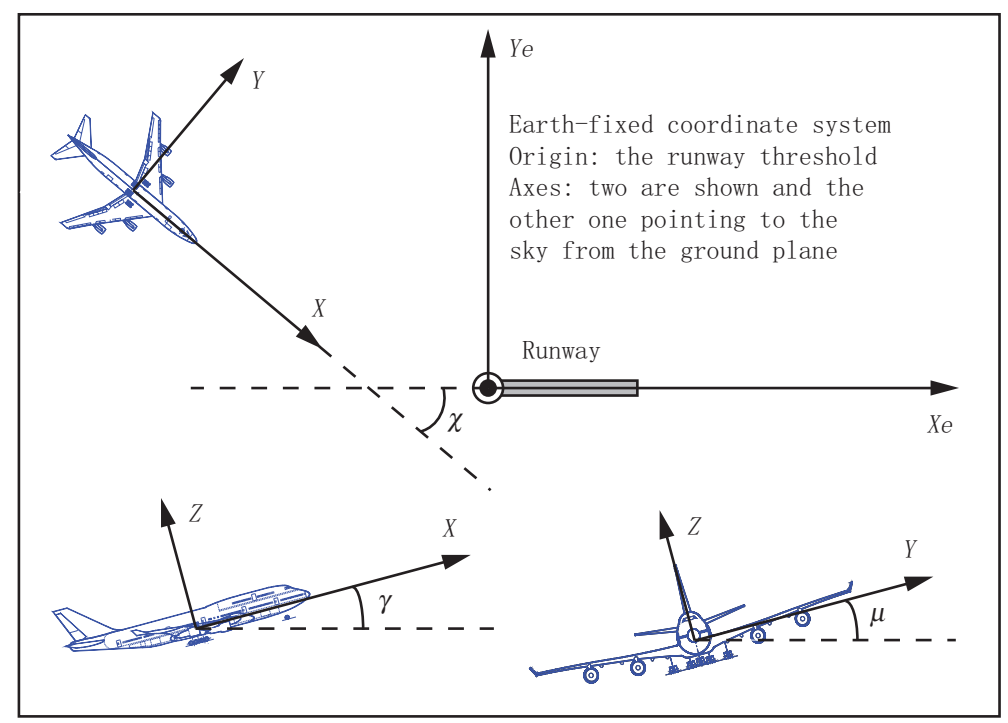

Figure 1. The Earth-fixed coordinate system and associated angles from different views.

Under all the assumptions and settings mentioned above, the point-mass equations of motion governing the movement of the aircraft have a general form of:

$$
\frac{\mathrm{d} \mathbf{x}}{\mathrm{d} t}=\mathbf{f}(\mathbf{x}(t), \mathbf{u}(t), t)
$$


in which $\mathbf{x}=\left[x, y, h, V_{T}, \gamma, \chi\right]^{T}$ is the state vector of the system and $\mathbf{u}=[\alpha, \mu, T]^{T}$ is the control vector into the system. The detailed expressions of Equation (2) are given by Equation (24) in the appendix. Both of the aerodynamic forces, $L$ and $D$ appearing on the right-hand side of the equations, are functions of $\alpha, h$ and, more importantly $V_{I}$. A relation between $V_{I}$ and $V_{T}$ is necessarily required to introduce $V_{I}$ in order to replace $V_{T}$ in the equations. In this work, the International Standard Atmosphere (ISA) model is employed so that the conversion ${ }^{11}$ from $V_{I}$ to $V_{T}$ has the form of:

$$
V_{T}=V_{I}\left[171233(288-0.006496 h)^{-2.128}\right] .
$$

Then the first derivative of $V_{T}$ with respect to $t$ can be written as

$$
\frac{\mathrm{d} V_{T}}{\mathrm{~d} t}=\left[171233(288-0.006496 h)^{-2.128}\right] \frac{\mathrm{d} V_{I}}{\mathrm{~d} t}+0.013823488 V_{I}\left[171233(288-0.006496 h)^{-3.128}\right] \frac{\mathrm{d} h}{\mathrm{~d} t} .
$$

By substituting Equation (3) into Equation (24), $V_{T}$ can be replaced by the appearances of $V_{I}$ and $h$. Moreover, with the assist of Equation (4), the fourth equation in Equation (24) can be rewritten into an equation with the derivative of $V_{I}$ with respect to $t$ on the left-hand side. By doing so, the general form of the equations of motion and the control vector hold out but $V_{T}$ is replaced by $V_{I}$ at any place in the equations. In order to step further to complete the reformulation, the chain rule is employed as follows:

$$
\frac{\mathrm{d} \mathbf{x}}{\mathrm{d} V_{I}}=\frac{\mathrm{d} \mathbf{x}}{\mathrm{d} t} \times \frac{\mathrm{d} t}{\mathrm{~d} V_{I}}
$$

Reformulation based on Equation (5) yields a new set of equations of motion with the general form of:

$$
\frac{\mathrm{d} \mathbf{x}}{\mathrm{d} V_{I}}=\mathbf{g}\left(\mathbf{x}\left(V_{I}\right), \mathbf{u}\left(V_{I}\right), V_{I}\right)
$$

with $\mathbf{x}=[x, y, h, t, \gamma, \chi]^{T}$ and $\mathbf{u}=[\alpha, \mu, T]^{T}$ as the state vector and control vector, respectively. The detailed expressions of Equation (6) are given by Equation (25) in the appendix.

\section{B. Optimal Control and Performance Index}

By definition, a history of the state variables during the time interval $\left[t_{i}, t_{f}\right]$ is call a "state history" and a history of the control variables during the same interval is called a "control history". In this work, the interval changes into $\left[V_{I, i}, V_{I, f}\right]$ but the names remain. In essence, $V_{I}$ correlates with $t$ in a one-to-one fashion. That is, the optimal control history from $t_{i}$ to $t_{f}$ can be obtained when the optimization routine is terminated. The objective of the optimization is to find the optimal control history from $V_{I, i}$ to $V_{I, f}$ to minimize (or maximize) a certain performance index. Clearly, different performance indexes lead to different optimal control histories and the resulted state histories are also different. To describe the noise impact of an arriving aircraft, candidates include noise contours at certain noise levels, complaints on noise from residential communities, sleep disturbance, etc. As mentioned in Section I, trajectories are designed by optimizing the distribution of noise on the ground around the airport. In order to do so, sleep disturbance on all residents in all the residential communities around the airport is selected as the performance index of the optimization.

Sleep disturbance is a major noise concern of the residential communities since sleep is essential for both physical and emotional health. Noise can make it difficult to fall asleep and it can also interfere with the sleepers to lower the quality of their sleep. Another reason of choosing sleep disturbance is that it is reasonable to expect all the residents to return home for sleep during the night and thus the distribution of possible victims of aviation noise can be obtained directly from census data. A main disadvantage of selecting sleep disturbance is that the extent to which aviation noise impacts on those residents varies from individual to individual. These variations make it difficult for researchers to obtain a fixed relationship between sleep disturbance and flight movements. However, in 1997, the Federal Interagency Committee on Aviation Noise (FICAN) predicted a conservative relationship ${ }^{12}$ between the percentage of the exposed residents to be behaviorally awakened (percent of awakenings) and the exposure to a single event (the indoor sound exposure level), as can be seen in Figure 2. This curve could be interpreted as predicting the maximum percent of the people expected to be behaviorally awakened by the single event. The curve is represented by the equation as follows

$$
P_{A, F}=0.0087\left(S E L_{i n}-30\right)^{1.79}
$$


where $P_{A, F}$ is the percent of awakenings and $S E L_{i n}$ is the indoor A-weighted sound exposure level. More recently, in 2008, the standard ${ }^{13}$ published by the American National Standard Institute (ANSI) and the Acoustical Society of American (ASA) presented another relationship written as

$$
P_{A, A}=\frac{100}{1+e^{-z}},
$$

with $z=-6.8884+0.04444 S E L_{i n}$. The curve can also be found in Figure 2. It is clear that Equation (7) is more conservative than Equation (8) and the former one better describes the upper bound of the data ${ }^{14-16}$ from field studies. In this work, the relationship given by the FICAN is employed to calculate the percent of awakenings and finally the number of awakenings as the performance index of the optimization.

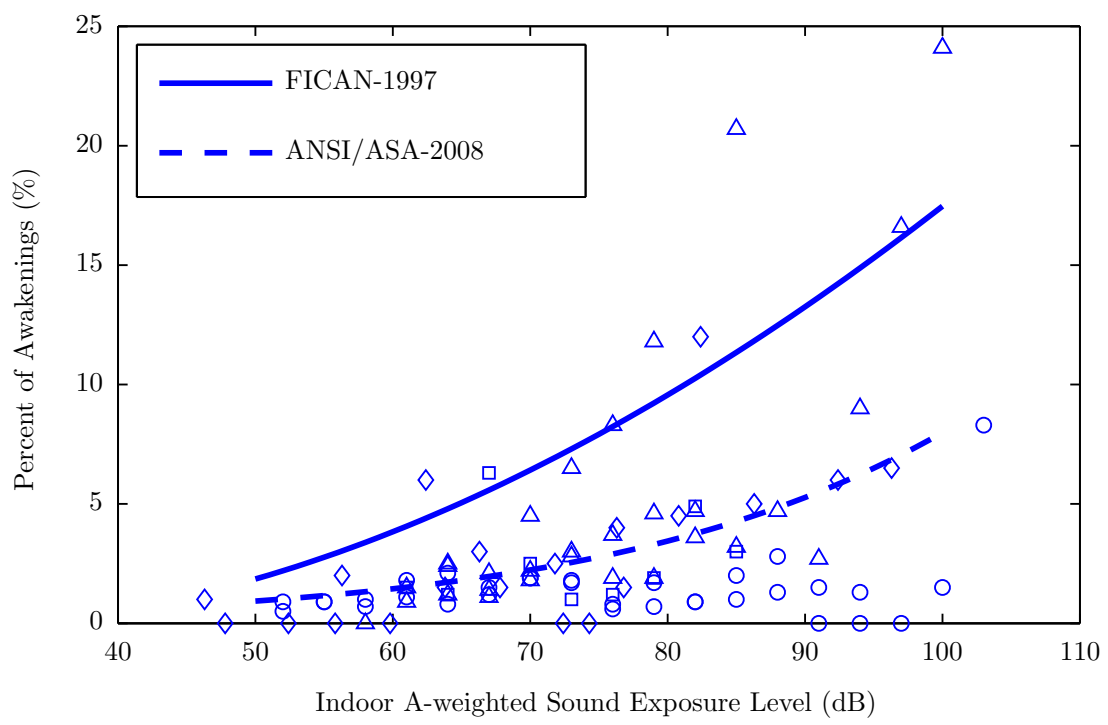

Figure 2. A plot of the percent of awakenings versus indoor sound exposure level.

\section{Optimization in A General Form}

The goal of the optimization is to minimize

$$
J\left(\mathbf{x}\left(V_{I}\right), \mathbf{u}\left(V_{I}\right)\right)
$$

subject to

$$
\mathbf{c}\left(\mathbf{x}\left(V_{I}\right), \mathbf{u}\left(V_{I}\right)\right) \leq 0
$$

in which $J$ is a scalar as the cost function of the optimization and $\mathbf{c}$ is a vector of all the constraints imposed on both the state and control variables. In short, an optimization is a smart and efficient search routine that tries to find the combination of $\mathbf{x}^{*}\left(V_{I}\right)$ and $\mathbf{u}^{*}\left(V_{I}\right)$ from the search domain defined by Equation (10) for which the value of the cost function is a minimum, denoted by $J^{*}$. This is an infinite-dimensional problem because the values of $\mathbf{x}\left(V_{I}\right)$ and $\mathbf{u}\left(V_{I}\right)$ at any instant value of $V_{I}$ have to specified.

\section{State Parameterization with Bernstein Polynomials}

This section summarizes the process of state parameterization using Bernstein polynomials. A number of independent state variables are parameterized and this parameterization process introduces a finite number of unknown parameters. The remaining state and control variables are written into functions of the introduced parameters based on Equation (6). In the end, the infinite-dimensional optimal control problem described by Equation (9-10) can be transformed into a finite-dimensional parametric optimization optimization problem. 


\section{A. Independent State Variables}

It is natural to treat control variables as independent variables and state variables as dependent ones, but it is not always necessary to do so. In certain cases, such as those involving derivatives of the state variables or terminal constraints on the state variables, it is relatively easy to treat some or all of the state variables as independent. In this work, the system is described by Equation (6) with initial and terminal values of $x, h$ and $y$. That is, derivatives of the state variables and terminal constraints on state variables are both involved. Therefore, state parameterization is employed to complete the transformation to obtain a finite-dimensional parametric optimization problem.

The crucial step is to select a set of independent state variables. Without losing generality, denote the dimension of the state vector as $m$ and the dimension of the control vector as $r$. Supposing that $r \leq m$, it is usually the case that the number of the independent state variables is $r$ and it is expected that the remaining $m-r$ state variables and $m$ control variables can be uniquely determined in terms of the selected independent state variables based on the state equations. As a matter of fact, one of the main drawbacks of state parameterization is that the determination of the independent state variables may be a cumbersome task and there is usually more than one way to do the selection if $r<m$. As for the problem dealt with in this work, there are six state variables and three control variables, meaning that three independent state variables have to be parameterized and the rest have to be determined through further derivation based on Equation (6). Considering that the trajectory is a three-dimensional curve which is always continuous and differentiable, $x\left(V_{I}\right), y\left(V_{I}\right)$ and $h\left(V_{I}\right)$ are three independent variables to be selected. The initial values of these variables are:

$$
\left\{\begin{array}{l}
x\left(V_{I, i}\right)=x_{i} \\
y\left(V_{I, i}\right)=y_{i} \\
h\left(V_{I, i}\right)=h_{i}
\end{array}\right.
$$

and the final values are:

$$
\left\{\begin{array}{l}
x\left(V_{I, f}\right)=x_{f} \\
y\left(V_{I, f}\right)=y_{f} \\
h\left(V_{I, f}\right)=h_{f}
\end{array} .\right.
$$

The trajectory to be optimized starts at $\left[x_{i}, y_{i}, h_{i}\right]$ and ends at $\left[x_{f}, y_{f}, h_{f}\right]$. The positions of the aircraft between these two points are parameterized by polynomials as functions of $V_{I}$.

\section{B. Bernstein Polynomials}

For a function $f(\tau)$ defined on the closed interval $[0,1]$, the Bernstein polynomial ${ }^{17}$ of order $n$ of $f(\tau)$ can be written into the following expression:

$$
B_{n}(\tau)=\sum_{i=0}^{n} f\left(\frac{i}{n}\right)\left(\begin{array}{c}
n \\
i
\end{array}\right) \tau^{i}(1-\tau)^{n-i},
$$

in which

$$
b_{i n}=\left(\begin{array}{c}
n \\
i
\end{array}\right) \tau^{i}(1-\tau)^{n-i}
$$

for $i=0, \ldots, n$ are called Bernstein basis polynomials. Evidently, $B_{n}(\tau)$ is a polynomial in $\tau$ of order less than or equal to $n$. Bernstein polynomials were first used in a constructive proof for the Weierstrass approximation theorem ${ }^{18}$. If $f(\tau)$ is continuous on the closed interval $[0,1]$, then we have:

$$
\lim _{n \rightarrow \infty} B_{n}(\tau)=f(\tau) .
$$

uniformly on $[0,1]$.

As for the practical optimization problem, a relation is first given as follows:

$$
V_{I}=V_{I, f}+\left(V_{I, i}-V_{I, f}\right)(1-\tau),
$$

from which it is easy to tell that $V_{I}$ decreases from $V_{I, i}$ to $V_{I, f}$ when $\tau$ increases from 0 to 1 . Meaning, if there is a function of $\tau$, it can also be written into a function of $V_{I}$ in a unique way based on Equation (16). 
Then, there Bernstein polynomials are given with the form of:

$$
\left\{\begin{aligned}
B_{1}\left(\tau, \mathbf{p}_{1}\right) & =\sum_{i=0}^{n} p_{1, i n} b_{i n}(\tau) \\
B_{2}\left(\tau, \mathbf{p}_{2}\right) & =\sum_{i=0}^{n} p_{2, i n} b_{i n}(\tau), \\
B_{3}\left(\tau, \mathbf{p}_{3}\right) & =\sum_{i=0}^{n} p_{3, i n} b_{i n}(\tau)
\end{aligned}\right.
$$

where $p_{1, i n}, p_{2, \text { in }}$ and $p_{3, \text { in }}$ are Bernstein coefficients from $\mathbf{p}_{1}, \mathbf{p}_{2}$ and $\mathbf{p}_{3}$, respectively. If all the Bernstein coefficients fall into the interval $[0,1]$, then the values of the Bernstein polynomials fall into the interval $[0,1]$ as well. The differences between the domain of the state variables and the proposed Bernstein polynomials desire further normalizations. For example, writing $x$ into a function of $B_{1}$ yields $x\left(B_{1}\left(\tau, \mathbf{p}_{1}\right)\right)$ which is actually $x\left(\tau, \mathbf{p}_{1}\right)$ and finally $x\left(V_{I}, \mathbf{p}_{1}\right)$. The same principle applies for $y$ and $h$ to finally obtain $y\left(V_{I}, \mathbf{p}_{2}\right)$ and $h\left(V_{I}, \mathbf{p}_{3}\right)$. As for the initial and final values of these state variables, there are equations as follows:

$$
\left\{\begin{array}{l}
x\left(V_{I, i}, \mathbf{p}_{1}\right)=x\left(B_{1}\left(0, \mathbf{p}_{1}\right)\right)=x_{i} \\
y\left(V_{I, i}, \mathbf{p}_{2}\right)=y\left(B_{2}\left(0, \mathbf{p}_{2}\right)\right)=y_{i} \\
h\left(V_{I, i}, \mathbf{p}_{3}\right)=h\left(B_{3}\left(0, \mathbf{p}_{3}\right)\right)=h_{i}
\end{array}\right.
$$

and

$$
\left\{\begin{array}{l}
x\left(V_{I, f}, \mathbf{p}_{1}\right)=x\left(B_{1}\left(1, \mathbf{p}_{1}\right)\right)=x_{f} \\
y\left(V_{I, f}, \mathbf{p}_{2}\right)=y\left(B_{1}\left(2, \mathbf{p}_{2}\right)\right)=y_{f} \\
h\left(V_{I, f}, \mathbf{p}_{3}\right)=h\left(B_{1}\left(3, \mathbf{p}_{3}\right)\right)=h_{f}
\end{array}\right.
$$

The search domain for each of these introduced parameters is the closed interval [0,1]. Equation (18) and Equation (19) are equality constraints on some of the parameters in $\mathbf{p}_{1}, \mathbf{p}_{2}$ and $\mathbf{p}_{3}$.

\section{Parametric Optimization Problem}

A summary of the state parameterization procedures is as follows:

1. Select the set of independent state variables;

2. Specify the parameterization and set a search domain for the introduced parameters; and

3. Determine the rest of the state variables and all control variables based on the state equation.

The first two steps have been finished and the third step requires lots of mathematical derivations. The result of the derivations is that $\gamma$ and $\chi$ from the state vector and all the variables in the control vector are written into functions of the introduced parameters. As an exception, the analytical expression of $t$ can not be derived but the terminal value of $t$ can still be known by numerical integration. Meaning, constraints on the flight time can still be imposed although there would not be any equalities as shown in Equation (18) and Equation (19). After executing all the procedures needed for state parameterization, the goal of the optimization changes into the minimization of

$$
J\left(V_{I}, \mathbf{p}_{1}, \mathbf{p}_{2}, \mathbf{p}_{3}\right)
$$

subject to

$$
\mathbf{c}\left(V_{I}, \mathbf{p}_{1}, \mathbf{p}_{2}, \mathbf{p}_{3}\right) \leq 0
$$

Clearly, the goal of the optimization routine is to find $\mathbf{p}_{1}^{*}, \mathbf{p}_{2}^{*}$ and $\mathbf{p}_{3}^{*}$ to obtain a minimum for the cost function, denoted again as $J^{*}$. This is an finite-dimensional problem because the number of unknown parameters is finite, which is the sum of the dimensions of $\mathbf{p}_{1}, \mathbf{p}_{2}$ and $\mathbf{p}_{3}$. 


\section{Models}

This section presents all the models needed in the optimization routine, including a Boeing 747-400 aircraft, a number of different population distribution models around the scheduled airport and the acoustic model of the selected aircraft. Note that the models are all replaceable in order to be able to cope with other similar problems. One can see that the models given below are quite specific due to the fact that they are all provided for the numerical calculations in Section $\mathrm{V}$ for the purpose of demonstration.

\section{A. Aircraft Model}

A Boeing 747-400 aircraft model ${ }^{9}$ is used in this work. According to the the flap extension speed schedule given in Table 1, the configuration of the aircraft changes while decelerating from $V_{I, i}$ to $V_{I, f}$. In practice there is a transition period for the flaps to be extended mechanically but in this work it is assumed that this change occurs instantaneously. The schedule is determined by the mass and the landing configuration of the aircraft. In this work, it is assumed that the mass of the aircraft is $260,000 \mathrm{~kg}$ and it remains constant due to the fact that the fuel consumption is small enough to be neglected. As for the configuration, it is set in such a way that the aircraft reaches the terminal point with Flaps 20 and landing gears extended. The flaps will further change to 25 degrees to establish the landing configuration but that part of the trajectory is not considered in the optimization routine.

Table 1. Flap extension speed schedule of a Boeing 747-400 aircraft.

\begin{tabular}{lccccc}
\hline \hline Flaps & $\mathrm{Up}$ & 1 & 5 & 10 & 20 \\
Gears & $\mathrm{Up}$ & $\mathrm{Up}$ & $\mathrm{Up}$ & $\mathrm{Up}$ & Down \\
\hline$V_{I}(\mathrm{kts})$ & $250 \rightarrow 218$ & $218 \rightarrow 198$ & $198 \rightarrow 178$ & $178 \rightarrow 158$ & $158 \rightarrow 148$ \\
\hline \hline
\end{tabular}

The aerodynamic performance of the aircraft changes when the configuration of the aircraft changes. Corresponding to different configurations, the expressions of $C_{L}$ as functions of $\alpha$ and the expressions of $C_{D}$ as functions of $C_{L}$ for the selected aircraft can be seen in Table 2. In essence, the expressions of $C_{D}$ can also be written into functions of $\alpha$. This is the reason why the aerodynamic lift and drag forces are both written as functions of $\alpha, h$ and $V_{I}$ in Equation (24) and Equation (25). In addition, it can be seen from Table 2 that there are both lower and upper bounds on $\alpha$ for each of the configurations, which are reflected by the constraints in Equation (21).

Table 2. Aerodynamic lift and drag coefficients of a Boeing 747-400 aircraft.

\begin{tabular}{cccccc}
\hline \hline Flaps & Gears & $\alpha$ & $C_{L}(\alpha)$ & $C_{L}$ & $C_{D}\left(C_{L}\right)$ \\
\hline $\mathrm{Up}$ & $\mathrm{Up}$ & {$[2.0,8.5]$} & $0.07500(\alpha+2)+0.025$ & {$[0.3250,0.8125]$} & $0.10370 C_{L}^{2}-0.0454 C_{L}+0.0233$ \\
1 & $\mathrm{Up}$ & {$[5.0,10.5]$} & $0.10000(\alpha+2)-0.200$ & {$[0.5000,1.0500]$} & $0.07180 C_{L}^{2}-0.0307 C_{L}+0.0299$ \\
5 & $\mathrm{Up}$ & {$[5.0,12.0]$} & $0.09500(\alpha+2)-0.065$ & {$[0.6000,1.2650]$} & $0.05710 C_{L}^{2}-0.0129 C_{L}+0.0344$ \\
10 & $\mathrm{Up}$ & {$[4.0,13.5]$} & $0.09750(\alpha+2)+0.035$ & {$[0.6200,1.5463]$} & $0.07260 C_{L}^{2}-0.0424 C_{L}+0.0463$ \\
20 & Down & {$[2.5,13.5]$} & $0.10000(\alpha+2)+0.250$ & {$[0.7000,1.8000]$} & $0.04696 C_{L}^{2}-0.0112 C_{L}+0.0723$ \\
\hline \hline
\end{tabular}

\section{B. Population Distribution Model}

It is neither realistic nor necessary to calculate the noise level exposed to each of the residents in the residential communities in the vicinity of the airport. In order to reduce the computational load, the vicinity of the airport is divided into a number of cells with regular or irregular shapes, named "regular grid" or "irregular grid". Each of these cell contains a number of residents and the cell generated in such a way that it is reasonable to assume that the noise levels exposed to all the residents in an individual cell are the same. 
Therefore, any point in the cell can be used as the observing point at which the A-weighted sound exposure level generated by the arriving aircraft is calculated. In practical cases, the cells usually have irregular shapes because they are probably generated by the shapes of these residential communities. In this work, however, the cells are all rectangles with the same size because the numerical examples given in Section $\mathrm{V}$ are just for the purpose of demonstration.

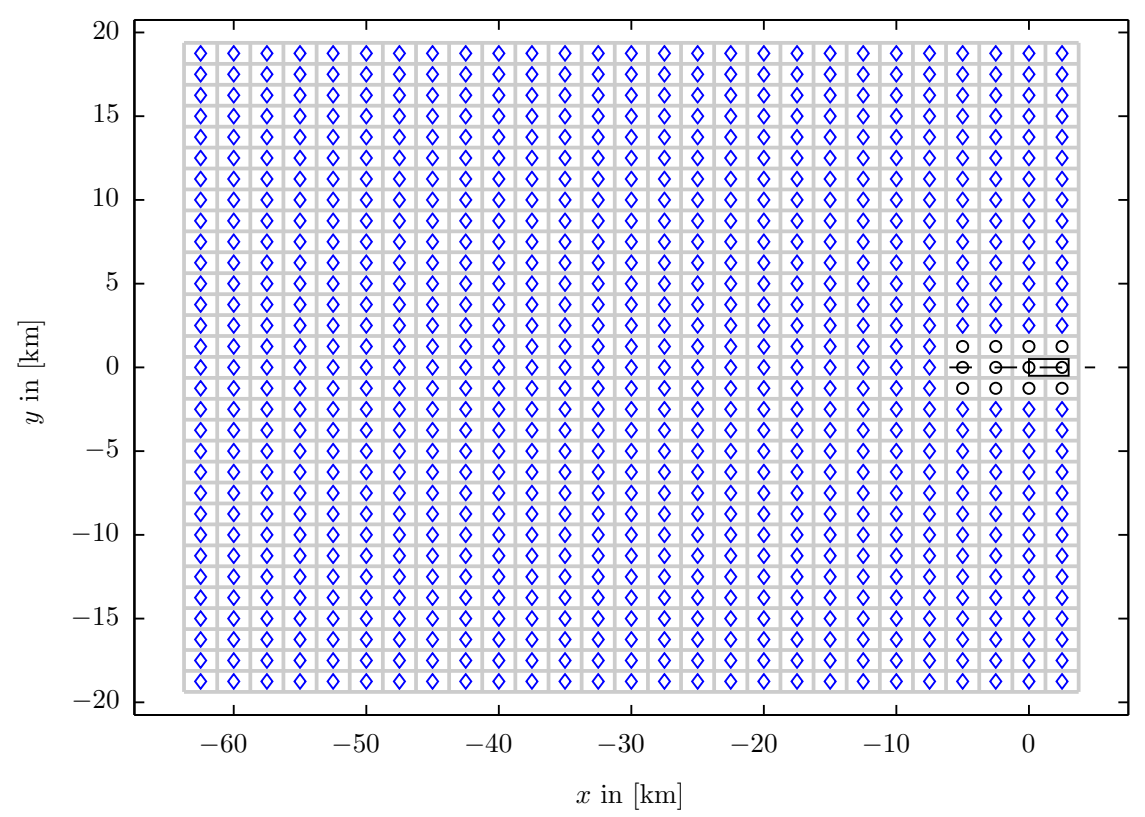

Figure 3. Uniformed population distribution to generate baseline results for comparisons.

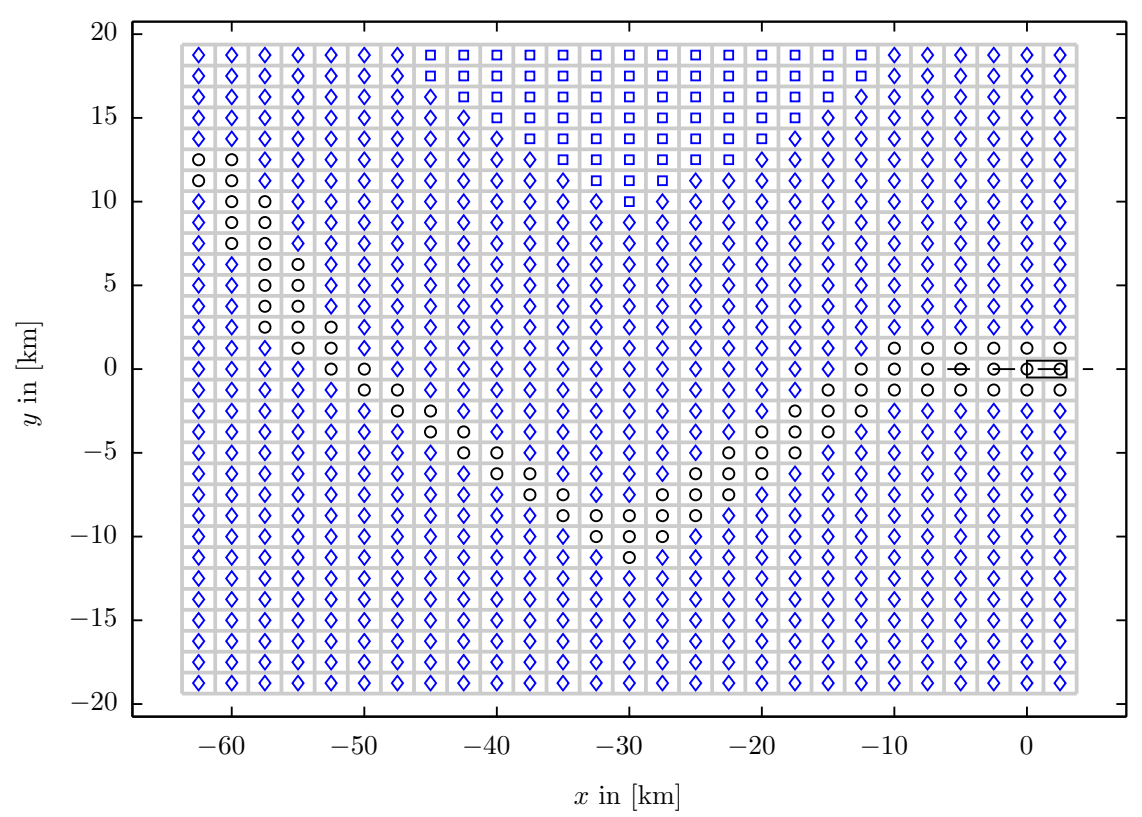

Figure 4. Specifically designed population distribution for optimization: case 1 


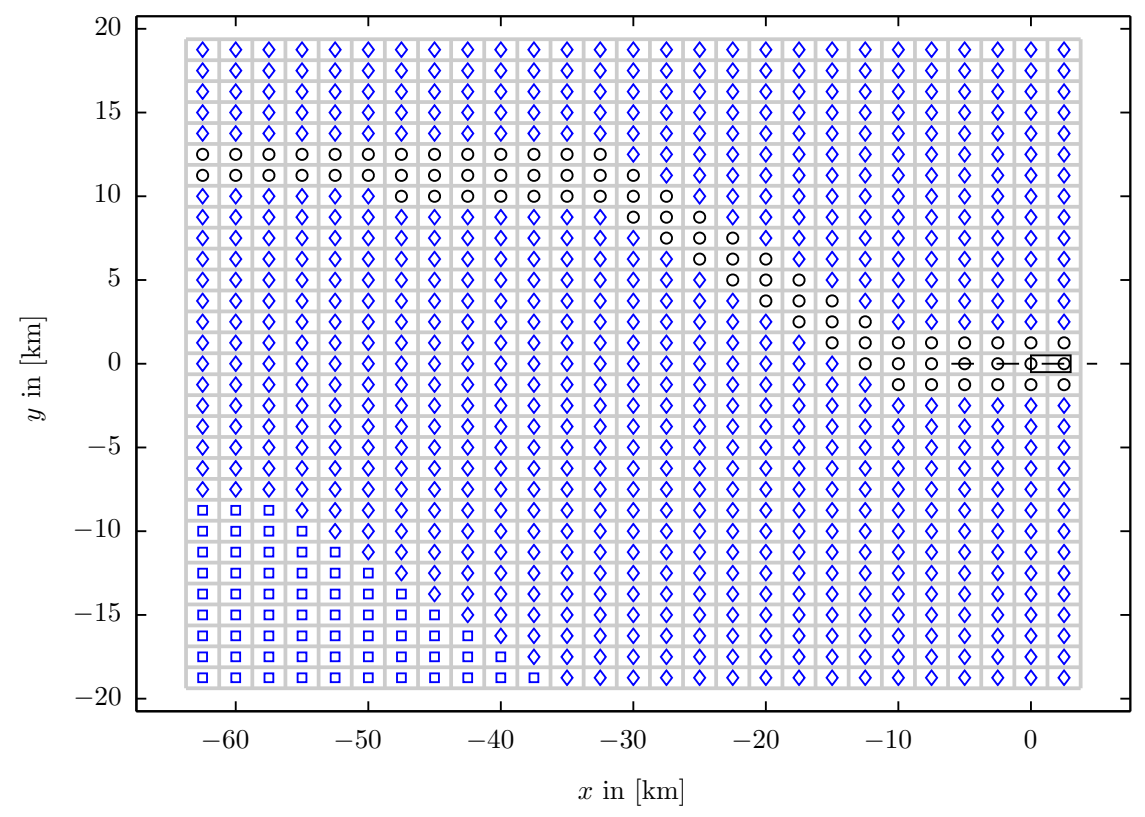

Figure 5. Specifically designed population distribution for optimization: case 2

As can be seen in Figure 3, a population distribution model is provided in order to generate baseline results to evaluate and analysis the optimization results that follow. Except for a number of zero-density cells surrounding the scheduled runway, the population density in each of the other cells is the same. This zero-density area remains in both models shown in Figure 6 and Figure 7. These two population distribution models are specifically designed, in which the residents are not distributed in a uniformed way any more. It can be seen from the figures that in each of them there is a route composed only by zero-density cells. In all these three models, the settings are following: (1) the black square represents the scheduled runway with its centerline plotted as a dashed line; (2) the gray rectangles represents cells with a size of $2.50 \mathrm{~km} \times 1.25$ $\mathrm{km}$; (3) all the observing points locate at the centers of the rectangles; (4) the "diamond" symbol represents that there are 3000 residents in the cell; (5) the "square" symbol represents that there are 6000 residents in the cell; and (6) the "circle" symbol represents that there are no residents at all.

\section{Acoustic Model}

For aircraft noise modeling, there exist a number of different methods, which can be mainly categorized into simulation methods and integrated methods. Each of these two groups has its own strengths and weaknesses. However, it is agreed that integrated methods represent the current best practice, especially in trajectory optimization problems where noise levels need be calculated in order to calculate the noise impact of a single event. The Aviation Environmental Design Tool (AEDT) is under development by the FAA. The AEDT is currently used by the U.S. government to consider the interdependencies between aircraft-related fuel burn, noise and emissions. AEDT is also being developed for public release and will become the next generation aviation environmental consequence tool, replacing the current public-use aviation air quality and noise analysis tools, such as the INM. Before that replacement happens, the method presented by the INM is used in this work. The INM has been a standard tool for the FAA since 1978 for determining the predicted noise impact in the vicinity of airports. The core of the method from the INM is the NPD datasets which are normalized to standard reference conditions ${ }^{19}$ in order to be most widely used for aircraft noise modeling. For conditions that are different from the reference, it is required to take into account appropriate corrections to offset the differences. One example dataset is given in Table 3 for the calculation of A-weighted sound exposure levels of the aircraft during approach. A flight trajectory has to be divided into a set of straight-line flight-path segments in order to calculate the outdoor A-weighted sound exposure levels using 
the NPD datasets. Knowing the appropriate geometry information of the flight-path segments, the noise level can either found directly from the datasets if the slant distance and the engine power are both tabulated in the tables or obtained using interpolation or extrapolation. Details can be found from a report given by the $\mathrm{ICAO}^{19}$ in 2008.

Table 3. NPD data for A-weighted sound exposure levels of a Boeing 747-400 aircraft installed with four PW4056 jet-engines during approach.

\begin{tabular}{ccccccccccc}
\hline \hline \multirow{2}{*}{$\begin{array}{c}\text { Engine Power } \\
{[\mathrm{lb}]}\end{array}$} & 200 & 400 & 630 & 1000 & 2000 & 4000 & 6300 & 10000 & 16000 & 25000 \\
\cline { 2 - 10 } & 102.9 & 98.2 & 94.9 & 91.5 & 86.1 & 80.1 & 75.6 & 70.5 & 65.2 & 60.1 \\
\hline \hline 1000 & 103.3 & 98.6 & 95.2 & 91.7 & 86.3 & 80.3 & 75.8 & 70.6 & 65.2 & 60.1 \\
13000 & 103.9 & 99.1 & 95.7 & 92.1 & 86.6 & 80.6 & 76.1 & 70.9 & 65.6 & 60.6 \\
16000 & 104.6 & 99.8 & 96.3 & 92.6 & 87.0 & 80.9 & 76.5 & 71.4 & 66.3 & 61.4 \\
\hline \hline
\end{tabular}

Furthermore, it is important to note that the goal is to calculate the indoor A-weighted sound exposure levels in order to obtain the percent of awakenings while the residents are sleeping. The reduction of the A-weighted sound exposure levels from outdoor to indoor is different when the windows are open or closed and it is also greatly dependent on the insulation of the house. In this work, it is assumed that the houses are averagely insulated and the reduction is set to be equal with $20.5 \mathrm{~dB}$.

\section{Numerical Examples}

Genetic algorithms are employed to solve the optimization problem formed above. With this kind of algorithms, it is expected to find the global optimum when the optimization routine is terminated by certain stopping criteria. For each of the numerical examples in this work, the same optimization routine is executed more than one time and the results are always different, indicating that it is even very hard to guarantee the global optimum for such a highly nonlinear optimization problem with multiple local optima with genetic algorithms. The results shown below are the best among all cases carried out by the authors.

\section{A. Baseline Result}

An optimal trajectory corresponding to the population distribution model shown in Figure 1 is obtained as the baseline result to be compared. As can be seen in Figure 1, given are the optimal trajectory and its projection on the ground. The coordinates of the blue star and the red star are:

$$
\left\{\begin{array}{l}
x_{i}=-57.869 \mathrm{~km} \\
y_{i}=12.000 \mathrm{~km} \\
h_{i}=10000 \mathrm{ft}
\end{array}\right.
$$

and

respectively.

$$
\left\{\begin{array}{ll}
x_{f}=-11.341 \mathrm{~km} \\
y_{f}= & 0 \mathrm{~km} \\
h_{f}= & 0 \mathrm{ft}
\end{array},\right.
$$

The first part of the trajectory is plotted into a red curve which corresponds to the configuration of "Flaps Up" and "Gears Up". Then the flaps are extended for the first time to establish the configuration of "Flaps 1" and "Gears Up", which happens at the start point of the yellow part of the trajectory. The indicated airspeed keeps on decreasing to reach the moment to extend the flaps further to 5 degrees and start flying along the blue curve instantaneously. The configuration is in fact "Flaps 5" and "Gears Up". The next configuration is established at the start point of the green part of the trajectory, which is "Flaps 10" and "Gears Up". Finally, the flaps are extended for the last time and the landing gears are engaged to establish the configuration of "Flaps 20" and "Gears Up", which occurs at the start point of the black curve. As for this optimal trajectory, the flight time is 398.4 seconds and the number of awakenings is 6347 . 


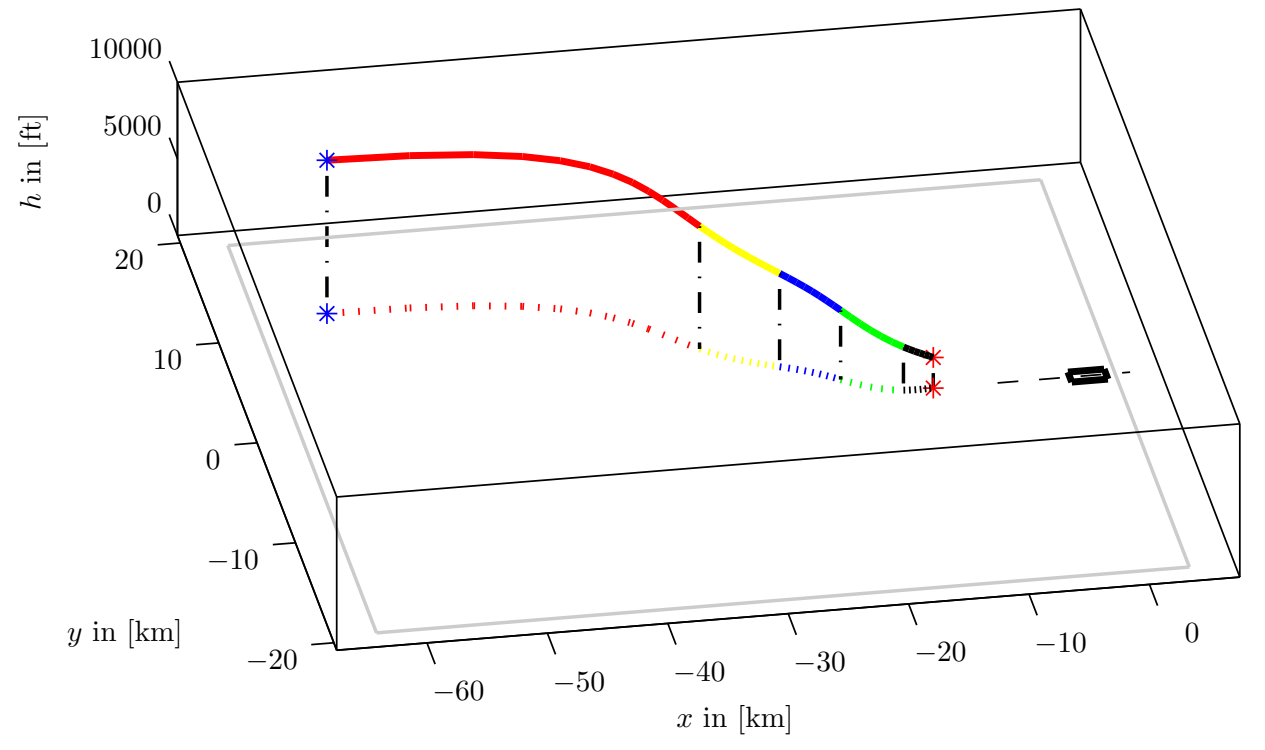

Figure 6. Optimal trajectory corresponding to the population distribution in Figure 1 as the baseline result.

\section{B. Specifically Designed Population Distribution: Case 1}

The trajectory starts at the point given in Equation (22) and ends at the point given in Equation (23). However, the population distribution is specifically designed by the authors with a zero-density route, as shown in Figure 2. The optimal trajectory and its projection on the ground are given in Figure 7.

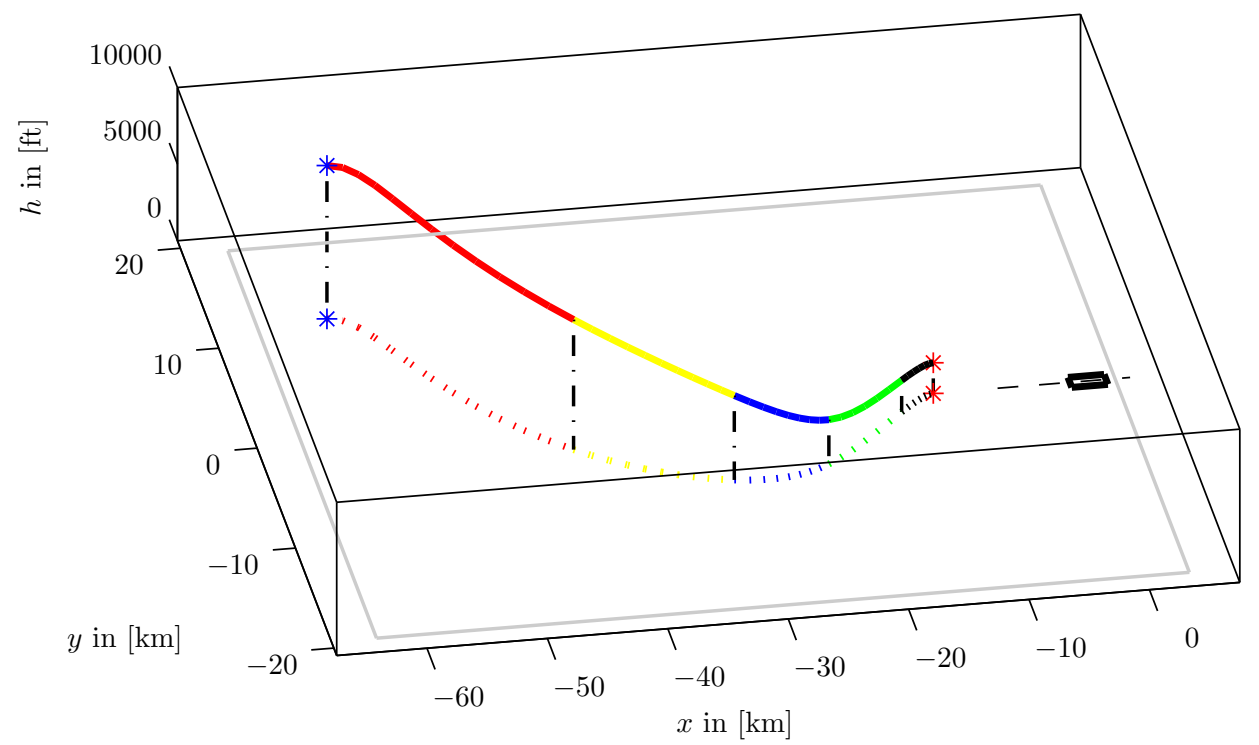

Figure 7. Optimal trajectory corresponding to the specifically designed population distribution in Figure 2.

It is easy to realize that the optimal trajectory is attracted by the zero-density route so that part of the trajectory is on the other side of the centerline of the runway. The curves with different colors have the same meanings as those mentioned previously. As for the optimal trajectory in this case, the flight time is 487.8 
seconds and the number of awakenings is 4799. Compared with the baseline result, the flight time increases by around $22.4 \%$ but the number of awakenings decreases by about $24.4 \%$.

\section{Specifically Designed Population Distribution: Case 2}

Again, the trajectory starts at the point given in Equation (22) and ends at the point given in Equation (23). The population distribution model employed in this example is the one given in Figure 3 . The optimal trajectory and its projection on the ground are given in Figure 8. Again, the optimal trajectory is attracted by the zero-density route which is specifically located and the curves with different colors have the same meanings as those mentioned previously. Another main difference between this optimal trajectory and the other two given in previous subsections is that the aircraft descends more steeply at the beginning and then takes a shallow descent to reach the red star. As for this optimal trajectory, the flight time is 433.8 seconds and the number of awakenings is 3588. Compared with the baseline result, the flight time increases by around $8.9 \%$ but the number of awakenings decreases by about $43.5 \%$.

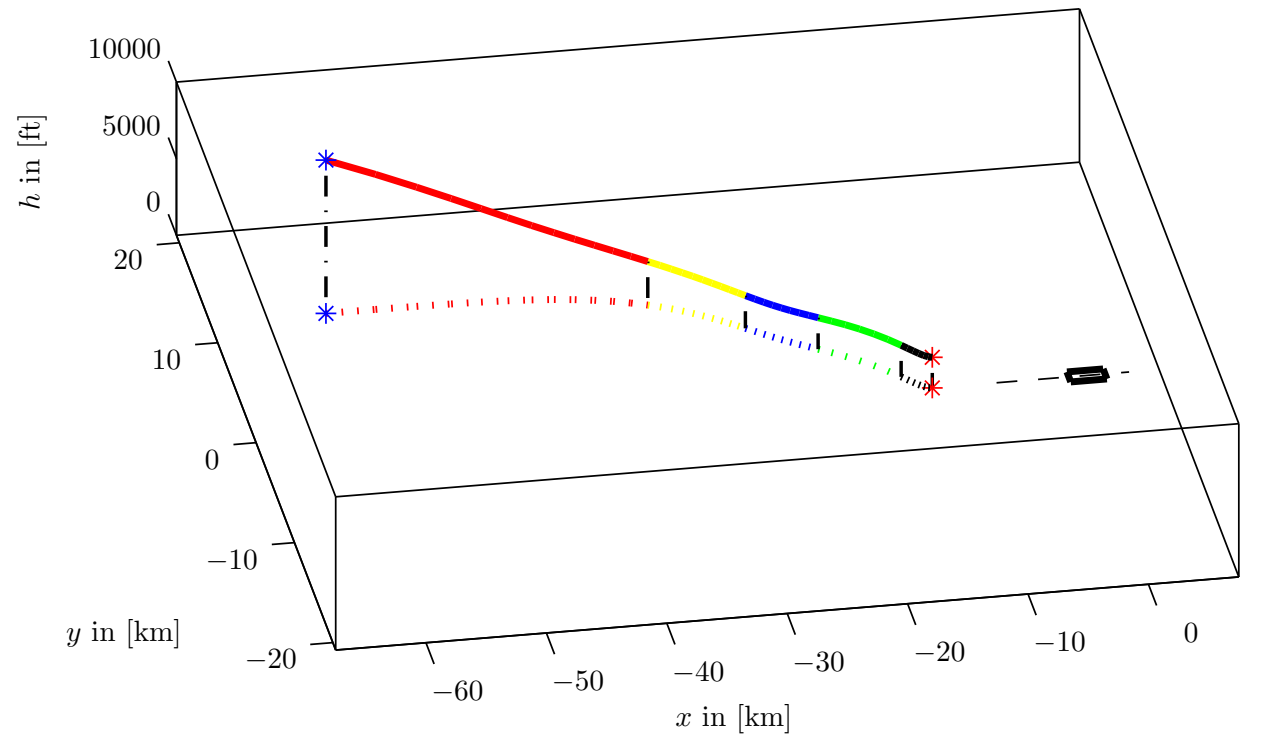

Figure 8. Optimal trajectory corresponding to the specifically designed population distribution in Figure 3.

\section{Discussions}

The number of residents enclosed in the area under consideration remains constants although they are distributed in different ways. That is the reason why the authors provide the first example as the baseline result. The results show that there are different optimal trajectories corresponding to different distribution situations of residents on the ground in the vicinity of the airport. These two specifically designed cases are a bit extreme because such zero-density routes barely exist in practice. Nevertheless, both of them function very well as demonstrations, indicating that the proposed optimization technique is able to find the optimal trajectory to generate the minimum number of awakenings.

\section{Conclusions}

In this work, a technique is developed to optimize the trajectory of an arriving passenger aircraft in order to minimize the noise impact generated within the entire phase of approach. Operations in the cockpit are taken into account by replacing the true airspeed by the indicated airspeed in the dynamic system. In order to do so, the conventional point-mass equations of motion are reformulated so that the derivatives in the formulas are with respect to the indicated airspeed instead of the flight time. Further, the 
infinite-dimensional optimal control problem is transformed into a finite-dimensional parametric optimization problem via state parameterization using Bernstein polynomials. The new optimization problem is solved using genetic algorithms. A set of numerical examples are provided to show that the proposed optimization technique is able to minimize the number of awakenings generated by an arriving aircraft, taking into the population distribution situation in the vicinity of the scheduled airport.

In future, the results presented in this work can be used as references to evaluate the performance of optimization algorithms developed based on interval analysis ${ }^{20-23}$. In theory, optimization using interval analysis guarantees the global optimum of the problem given by Equation (20) and Equation (21). Results will be given in following publications.

\section{Appendix}

Corresponding to Equation (1), the conventional point-mass equations of motion governing an aircraft moving in a 3-dimensional space are given by:

$$
\left\{\begin{aligned}
\frac{\mathrm{d} x}{\mathrm{~d} t} & =V_{T} \cos \gamma \cos \chi \\
\frac{\mathrm{d} y}{\mathrm{~d} t} & =V_{T} \cos \gamma \sin \chi \\
\frac{\mathrm{d} h}{\mathrm{~d} t} & =V_{T} \sin \gamma \\
\frac{\mathrm{d} V_{T}}{\mathrm{~d} t} & =\frac{T-D\left(\alpha, h, V_{T}\right)-m g \sin \gamma}{m} \\
\frac{\mathrm{d} \gamma}{\mathrm{d} t} & =\frac{L\left(\alpha, h, V_{T}\right) \cos \mu-m g \cos \gamma}{m V_{T}} \\
\frac{\mathrm{d} \chi}{\mathrm{d} t} & =\frac{L\left(\alpha, h, V_{T}\right) \sin \mu}{m V_{T} \cos \gamma}
\end{aligned}\right.
$$

where $\mathbf{x}=\left[x, h, y, V_{T}, \gamma, \chi\right]^{T}$ and $\mathbf{u}=[\alpha, \mu, T]^{T}$ are the state vector and control vector, respectively.

Corresponding to Equation (6), the point-mass equations of motion governing an aircraft moving in a 3-dimensional space after reformulation are written as:

$$
\left\{\begin{aligned}
\frac{\mathrm{d} x}{\mathrm{~d} V_{I}} & =\frac{m V_{I}\left[171233(288-0.006496 h)^{-2.128}\right]^{2} \cos \gamma \cos \chi}{T-D\left(\alpha, h, V_{I}\right)-m g \sin \gamma-0.013823488 m V_{I}^{2}\left[171233^{2}(288-0.006496 h)^{-5.128}\right] \sin \gamma} \\
\frac{\mathrm{d} y}{\mathrm{~d} V_{I}} & =\frac{m V_{I}\left[171233(288-0.006496 h)^{-2.128}\right]^{2} \cos \gamma \sin \chi}{T-D\left(\alpha, h, V_{I}\right)-m g \sin \gamma-0.013823488 m V_{I}^{2}\left[171233^{2}(288-0.006496 h)^{-5.128}\right] \sin \gamma} \\
\frac{\mathrm{d} h}{\mathrm{~d} V_{I}} & =\frac{m V_{I}\left[171233(288-0.006496 h)^{-2.128}\right]^{2} \sin \gamma}{T-D\left(\alpha, h, V_{I}\right)-m g \sin \gamma-0.013823488 m V_{I}^{2}\left[171233^{2}(288-0.006496 h)^{-5.128}\right] \sin \gamma} \\
\frac{\mathrm{d} t}{\mathrm{~d} V_{I}} & =\frac{m\left[171233(288-0.006496 h)^{-2.128}\right]}{T-D\left(\alpha, h, V_{I}\right)-m g \sin \gamma-0.013823488 m V_{I}^{2}\left[171233^{2}(288-0.006496 h)^{-5.128}\right] \sin \gamma} \\
\frac{\mathrm{d} \gamma}{\mathrm{d} V_{I}} & =\frac{\frac{L\left(\alpha, h, V_{I}\right) \cos \mu-m g \cos \gamma}{T-D\left(\alpha, h, V_{I}\right)-m g \sin \gamma-0.013823488 m V_{I}^{2}\left[171233^{2}(288-0.006496 h)^{-5.128}\right] \sin \gamma}}{\frac{L\left(\alpha, h, V_{I}\right) \sin \mu}{V_{I} \cos \gamma}} \\
\frac{\mathrm{d} \chi}{\mathrm{d} V_{I}} & =\frac{V_{I}}{T-D\left(\alpha, h, V_{I}\right)-m g \sin \gamma-0.013823488 m V_{I}^{2}\left[171233^{2}(288-0.006496 h)^{-5.128}\right] \sin \gamma}
\end{aligned}\right.
$$

where $\mathbf{x}=[x, h, y, t, \gamma, \chi]^{T}$ is the newly selected state vector and the control vector remains $\mathbf{u}=[\alpha, \mu, T]^{T}$. 


\section{Acknowledgments}

This work is finished under the framework of CleanEra, a project initiated and supported by the Faculty of Aerospace Engineering at Delft University of Technology in The Netherlands.

\section{References} 1999.

${ }^{1}$ Berglund, B., Lindvall, T., and Schwela, D. H., "Guidelines for Community Noise," Tech. rep., World Health Organization,

${ }^{2}$ ICAO, "Annex 16 to the Convention on International Civil Aviation, Environmental Protection, Volume I, Aircraft Noise, Fifth Edition," Tech. rep., International Civil Aviation Organization, 2008.

${ }^{3}$ ICAO, "Guidance on the Balanced Approach to Aircraft Noise Management, Second Edition," Tech. rep., International Civil Aviation Organization, 2008.

${ }^{4}$ Koeslag, M. F., "Advanced Continuous Descent Approaches - An Algorithm Design for the Flight Management System," Tech. Rep. NLR-TR-200103142, National Aerospace Laboratory, 2001.

${ }^{5}$ Hull, D. G., "Conversion of Optimal Control Problems into Parameter Optimization Problems," Journal of Guidance, Control and Dynamics, Vol. 20, No. 1, 1997, pp. 57-60.

${ }^{6}$ von Stryk, O. and Rulirsch, R., "Direct and Indirect Methods for Trajectory Optimization," Annals of Operations Research, Vol. 37, 1992, pp. 357-373.

${ }^{7}$ Sirisena, H. R. and Chou, F. S., "State parameterization approach to the solution of optimal control problems," Optimal Control Applications and Methods, Vol. 2, 1981, pp. 289-298.

${ }^{8}$ Mehne, H. H. and Borzabadi, A. H., "A numerical method for solving optimal control problems using state parameterization," Numerical Algorithms, Vol. 42, 2006, pp. 165-169.

${ }^{9}$ Teengs, M., "Model of the Boeing 747-400 with CF6-80C2B1F Engines," Tech. rep., Delft University of Technology, 2003.

${ }^{10}$ Boeker, E. R., Dinges, E., He, B., Fleming, G., Roof, C. J., Gerbi, P. J., Rapoza, A. S., and Hemann, J., Integrated Noise Model (INM) Version 7.0 Technical Manual, Federal Aviation Adminstration, 2008, FAA-AEE-08-01.

${ }^{11}$ ICAO, "Procesures for Air Navigation Services," Tech. rep., International Civil Aviation Organization, 2006.

${ }^{12}$ FICAN, "Effects of Aviation Noise on Awakenings from Sleep," Tech. rep., Federal Interagency Committee on Aviation Noise, 1997.

13 "Quantities and Procedures for Description and Measurement of Environmental Sound - Part 6: Methods for Estimation of Awakenings Associated with Outdoor Noise Events Heard in Homes," .

${ }^{14}$ Fidell, S., Pearsons, K., Tabachnick, B., Howe, R., Silvati, L., and Barber, D., "Fied Study of Noise-Induced Sleep Disturbance," Journal of Acoustic Society of America, Vol. 98, 1995, pp. 1025-1033.

${ }^{15}$ Fidell, S., Howe, R., Tabachnick, B., Pearsons, K., and Sneddon, M., "Noise-Induced Sleep Disturbance in Residences Near Two Civil Airports," Tech. rep., National Aeronautics and Space Administration, 1995.

${ }^{16}$ Pearsons, K., Barber, D., Tabachnick, B., and Fidell, S., "Predicting Noise-Induced Sleep Disturbance," Journal of Acoustic Society of America, Vol. 97, 1995, pp. 331-338.

${ }^{17}$ Lorentz, G. G., Bernstein Polynomials, Chelsea Publishing Company, 1986.

${ }^{18}$ Gzyl, H. and Palacios, J. L., "The Weierstrass Approximation Theorem and Large Deviations," The American Mathematical Monthly, Vol. 104, 1997, pp. 650-653.

${ }^{19}$ ICAO, "Recommended Method for Computing Noise Contours Around Airports, First Edition," Tech. rep., International Civil Aviation Organization, 2008.

${ }^{20}$ Hansen, E. R., "Global Optimization using Interval Analysis - The One Dimensional Case," Journal of Optimization Theory and Applications, Vol. 29, No. 3, 1979, pp. 331-344.

${ }^{21}$ Hansen, E. R., "Global Optimization using Interval Analysis - The Multi-Dimensional Case," Journal of Optimization Theory and Applications, Vol. 34, No. 3, 1980, pp. 247-270.

${ }^{22}$ Hansen, E. R., Global Optimization using Interval Analysis, Marcel Dekker, Inc., 1992.

${ }^{23}$ Hansen, E. R. and Walster, G. W., Global Optimization using Interval Analysis: Second Edition, Revised and Expanded, Marcel Dekker, Inc. and Sun Microsystems, Inc., 2004. 